\title{
Microwave - ultrasound combined reactor suitable for atmospheric sample preparation procedure of biological and chemical products
}

\author{
A. Lagha ${ }^{1}$, S. Chemat ${ }^{1,2}$, P.V. Bartels ${ }^{2}$ and F. Chemat ${ }^{2, *}$ \\ ${ }^{1}$ Institut de Chimie Industrielle, University of Blida, BP. 270, route de Soumaa, 09000 Blida, Algeria \\ ${ }^{2}$ Agrotechnological Research Institute ATO-DLO, P.O. Box 17, NL- 6700 AA Wageningen, The Netherlands
}

\begin{abstract}
A compact apparatus in which a specific position can be irradiated by microwaves (MW) and ultrasound (US) simultaneously has been developed. The MW-US reactor has been designed for atmospheric pressure digestion and dissolution of biological and chemical products. The reactor can treat a range of throughputs of 0.1 to 10 grams of a liquid or solid sample. Its application in analytical chemistry has been shown by two examples where decreases in digestion and dissolution time were demonstrated: determination of copper in olive oil and dissolution of refractory mineral material $\mathrm{Co}_{3} \mathrm{O}_{4}$. Simultaneous microwave and ultrasound irradiation is shown as a new technique for atmospheric pressure digestion and dissolution of solid and liquid samples suitable for chemical and biological analysis.
\end{abstract}

Key words. Microwave - ultrasound - olive oil - mineral - digestion - dissolution.

\section{Introduction}

Nowadays, sample preparation is recognised as an important step in analytical chemistry. It is time consuming and an important source of errors and contamination. Therefore, the complete digestion of the sample is required to achieve reproducible and accurate elemental results with instrumental analytical methods [1-4]. In the past few years, sample preparation techniques such as solid phase extraction or supercritical fluid extraction have become a long way in satisfying analytical chemists needs for dissolution samples that can be introduced directly into analytical instruments. Analysts are exploring new techniques actually, and older techniques with new twists are receiving more attention [5-7].

In general, an analytical procedure comprises two steps: digestion (dissolution or extraction) and analysis (CPG, HPLC, AAS...). Whereas the last step is finished after only 15 minutes, digestion takes at least several hours. It is frequently done by a prolonged heating and stirring in strong acid solution. Microwave [8,9] and ultrasound [10-12] have been used to remedy these constraints: reduction of digestion time, reduction of the quantity of reagents, reduction of contamination, total automation and more safety. This explains the recent interest for these new methods by chemists and analysts and the large number of research articles and patents.

Whereas, commercially available microwave ovens and ultrasound baths have been introduced into analytical labo- ratories to achieve fast sample dissolution, combination of MW-US has not been applied or studied for digestion or extraction yet. The only available references [13-15] discussing simultaneous irradiation by microwaves and ultrasound are in the field of organic chemistry applications. These articles reveal the need for models that account for this coupled interaction of heat and mass transport, particularly models that take account about the specificity of each radiation MW and US and can be useful for analytical chemistry, extraction and synthesis.

The purpose of this study was to develop a rapid, reliable and effective digestion and dissolution method using a microwave oven equipped with a specific ultrasound probe. This method was compared with classical and microwave digestion procedures and various experimental conditions were tested for their effect on digestion efficiency to determine the optimum and limiting conditions for use of the MW-US combination in digestion, dissolution and extraction processes.

In this paper, we describe the kinetic comparative study of dissolution of refractory mineral material $\mathrm{Co}_{3} \mathrm{O}_{4}$ (mineral taken as a model system) in nitric acid. A study of the effect of various reactions conditions (microwave and ultrasound power, concentration, and reaction time) on the combined MW-US digestion is given. Finally, the combined MW-US method for is applied to olive oil (model of food products) for copper analysis with electrothermal atomic absorption spectrometry (ETAAS). 


\section{Theoretical}

\section{Microwave heating}

In recent decades microwave heating has taken an undeniable place in analytical and organic laboratories practice as a very effective and non-polluting method of activation. Examples of this technology in digestion and organic synthesis are numerous [1]. Microwaves are electromagnetic waves with a frequency range from $100 \mathrm{MHz}$ to $3 \mathrm{GHz}$. MW are composed of an electric and magnetic field and thus represent electromagnetic energy. This energy can act as a nonionising radiation that causes molecular motions of ions and rotation of the dipoles, but does not effect molecular structure.

The heat generation in the sample that is obtained with microwave energy requires the presence of dielectric compounds. The rotation of the dipoles in an alternating field causes friction, which produces heat. More precisely, the applied microwave field causes the molecules, on average, to temporarily spend slightly more time orienting themselves in the direction of the electric field rather than in other directions. When the field is removed, thermal agitation returns the molecules to a disordered state in the relaxation time and thermal energy is released. Microwave assisted digestion, dissolution or extraction constitute a thriving field gathering the thermal effects of microwaves (reversed heat transfer and activation of solid's surface) and their chemical effects (dielectric polarisation).

In conventional heating heat transfers occur from the heating device to the medium, while in microwave heating heat is dissipated inside the irradiated medium. MW is a mass heating, and heat transfers occur from the treated medium to the outside. This induces another important difference. In conventional heating heat transfers depend on thermal conductivity, on the temperature difference across the material, and on convection currents, this last term being quite often the most important. As a result the temperature increase is often rather slow, while in microwave heating, due to the mass heating effect, much faster temperature increases can be obtained, depending on the microwave power and the loss factor of the material being irradiated. But it is also well known that microwave field distribution is not homogeneous in the irradiated material; therefore energy is also not homogeneously dissipated, and "hot spots" occur if heat generation is faster than heat transfers. This problem is also connected with penetration depth, $D_{\mathrm{p}}=\lambda_{0} \varepsilon^{\prime} / 2 \pi \varepsilon^{\prime \prime}$, the depth at which energy is reduced to $1 / \mathrm{e}$ of original intensity $\left(\lambda_{0}=\right.$ wave length, $\varepsilon^{\prime}=$ dielectric constant, $\varepsilon^{\prime \prime}=$ loss factor). Hot spots have actually been observed in poorly conductive materials (solids or highly viscous media), but could possibly occur in liquids, as such "hot spots" have been observed at the surface of boiling liquids by IR measurements [16].

\section{Ultrasound cavitation}

Power ultrasound, having frequencies between $20 \mathrm{kHz}$ and $1 \mathrm{MHz}$, are now well known to have significant effects on the rate of various processes in the analytical laboratory. Cleaning and solubilisation are the more developed application and a large variety of ultrasound baths exist for chemical laboratory use. Extraction, exploiting the effect of the wave on a solid, is widely used for the extraction of essential oils from plant materials or metal impurities from soils and wines. Degassing and stripping, appropriate to the elimination of gas or recuperation of odours and flavours, are used in environmental and polymer laboratories. Other ultrasound applications are interesting: homogenisation, emulsification, sieving, filtration, and crystallisation. The most interesting effect of operational units is a reduction of the processing time and an increase of the product quality [17].

All these effects are attributed to acoustic cavitation: when a liquid is irradiated by ultrasound, microbubbles can appear, grow and oscillate extremely fast, and even collapse violently if the acoustic pressure is high enough. These collapses, occurring near a solid surface, will generate microjets and shockwaves, resulting in cleaning and erosion of the surface, and fragmentation of the solid. Moreover, in homogeneous liquid phase, the mechanism can be explained by two competing theories. The hot spot theory assumes that high pressures and temperatures generated in the bubbles during the last nearly adiabatic compression, just before collapse, are responsible for the breakage of molecular bonds and formation of radicals. On the other hand, the electrical theory involves micro-discharges due to high electrical fields generated by deformation and fragmentation of the bubbles [18].

\section{Combination MW-US}

The combination of these two types of irradiation, electromagnetic $(2.45 \mathrm{GHz})$ and mechanical $(20 \mathrm{kHz})$, and their application to physical processes like digestion, dissolution and extraction appears interesting. But, it is not known how simultaneous MW-US irradiation brings about a physical effect. Therefore, it is expected that not only the kinetic of the digestion would be increased but probably also a new effect would occur. For instance, the high energy level of a bubble cavitation under ultrasound can induce particle fragmentation and molecule excitation, and microwave polarisation can induce dielectric volumetric heating and selective heating of solid particles. A comparison between ultrasound (cavitation phenomena) and microwave (dielectric heating) is illustrated in figure 1.

\section{Material and methods}

\section{Apparatus}

The apparatus is illustrated in figure 2. It is based on a Prolabo Maxidigest 350 monomode microwave oven cavity operating at $2.45 \mathrm{GHz}$ with a power range from 0 to 300 watts. The borosilicate reactor is an open vessel operating at atmospheric pressure and has a capacity of 20 to $150 \mathrm{~mL}$. The incident microwave power is controlled with a 


\section{Original articles}
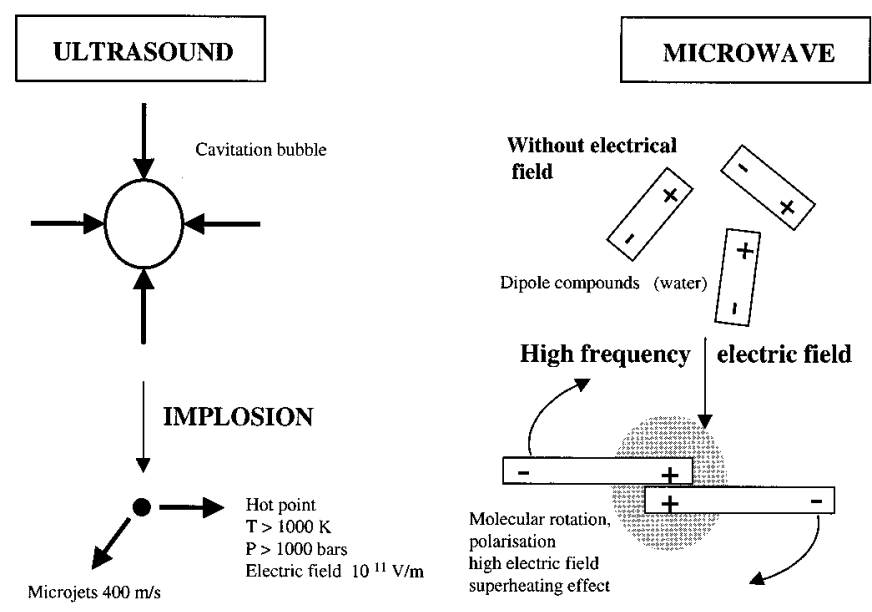

Figure 1. Analogy between ultrasound cavitation and microwave heating.

programmer which allows to work with one or several steps of microwave incident power according to the duration expressed in minutes. The ultrasound system is a cup horn type Branson Sonifier 250 (diameter of the transducer's tip $18 \mathrm{~mm})$. The emission of the ultrasound waves $(20 \mathrm{kHz})$ is made at the bottom of the reactor. The ultrasound probe is not in direct contact with the reactive mixture.

It is placed a distance from the electromagnetic field in order to avoid interactions and short circuits. The propagation of the ultrasound waves in the reactor is made by means of decaline introduced into the double jacket $(200 \mathrm{~mL})$. This liquid was chosen because of its low viscosity which induces good propagation of ultrasound and its inertia towards microwaves.

\section{Reagents, samples and glassware}

Nitric acid 98\%, (Prolabo).

Hydrogen peroxide 33\%, (Prolabo).

$\mathrm{Co}_{3} \mathrm{O}_{4}(99 \%+)$ Fluka.

The olive oil used for the recovery experiments was obtained from a local grocery.

All glassware was scrupulously cleaned and acid-soaked prior to use.

\section{Analysis equipment}

The concentration of cobalt and copper in the leaching solutions were determined by electrothermal atomic absorption spectrometry (ETAAS: Perkin Elmer AAnalyst 300).

\section{Classical procedure}

All the digestions were carried out using an electrical heating block "Büchi 430". Olive oil and $\mathrm{Co}_{3} \mathrm{O}_{4}$ were digested by nitric acid and hydrogen peroxide. The experiments were

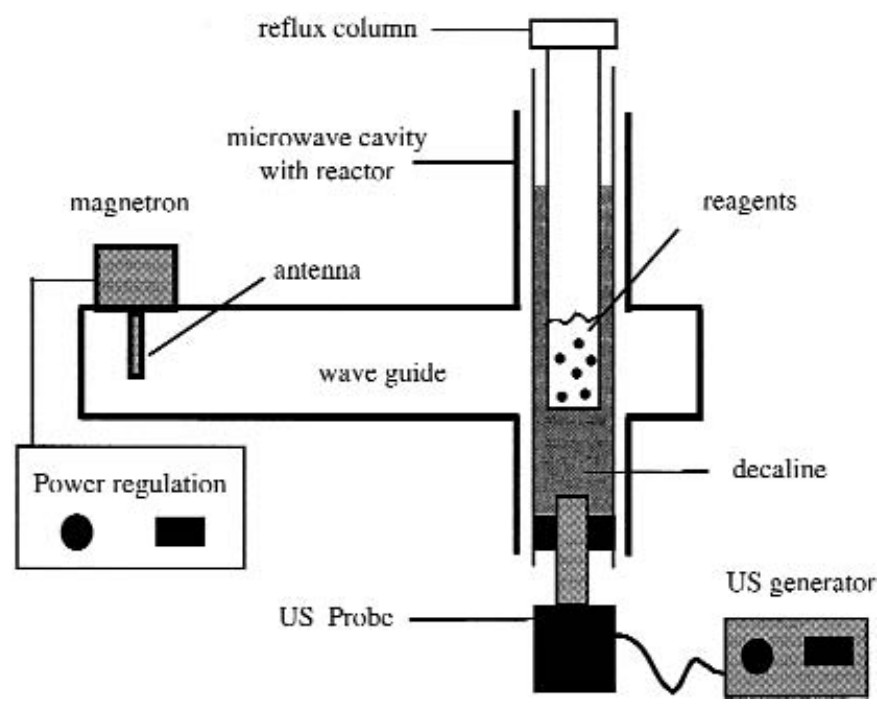

Figure 2. Schematic drawing of the single mode microwave system combined with ultrasound.

carried out at atmospheric pressure. Finally, ETAAS analysis gave the concentration of the analysed compounds. The digestion is the principal phase in the protocol and takes several hours. The success of the analysis depends on the efficiency of this digestion process.

It must be conducted with the highest rigour. The degree of copper or cobalt reported in this study is given from average of three identical tests.

\section{Microwave procedure}

The sample was mixed with $20 \mathrm{~mL}$ of nitric acid without heating. Then, microwave charring of the organic matrix was carried out. The charring duration represents $80 \%$ of the digestion or dissolution time. After that, hydrogen peroxide was added without heating. Microwave oxidation permits the destruction of the most resistant molecules. This step represents only $20 \%$ of the total duration. A microwave digestion protocol was adopted from several scientific works in this field [19-22] and presented in figure 3. Procedure A $\left(\mathrm{Co}_{3} \mathrm{O}_{4}\right)$ : microwave power $P_{\text {char }}=150 \mathrm{~W}$, time $t_{\text {char }}=1-6$ hours; $P_{\text {oxi }}=290 \mathrm{~W}, t_{\text {oxi }}=5-15 \mathrm{~min}$. Procedure B (olive oil): $P_{\text {char }}=180 \mathrm{~W}, t_{\text {char }}=30 \mathrm{~min} ; P_{\text {oxi }}=290 \mathrm{~W}, t_{\text {oxi }}=$ 3 min.

\section{Safety considerations}

Microwave and ultrasound processes are simple and can be readily understood in terms of the operating steps to be performed. However, the application of microwave energy to strong acids and oxidants, such as $\mathrm{HNO}_{3}$ or $\mathrm{H}_{2} \mathrm{O}_{2}$, can pose serious hazards in inexperienced hands. Given the fact that chemical and physical principles underlying the technology are not simple. An extraordinary level of safety and 


\section{Original articles}

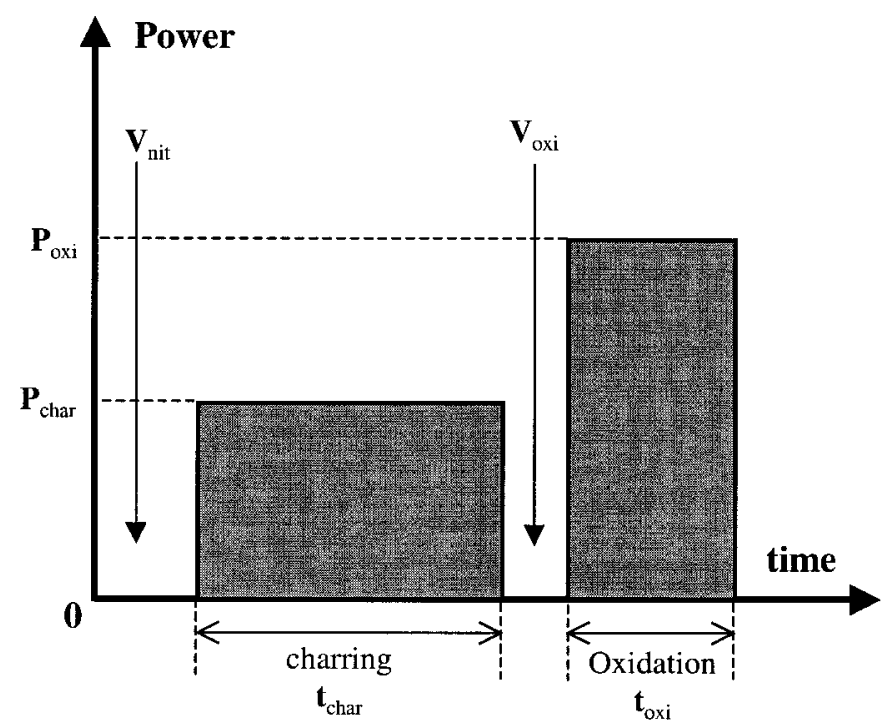

Figure 3. Standard description of atmospheric microwave digestion.

attention to details when planing and performing experiments must be used by all the persons dealing with microwaves. They have to ensure that they seek proper information from knowledgeable sources and that they do not attempt to implement these techniques unless proper guidance is provided. Only approved equipment and scientifically sound procedures should be used at all the times $[1,23]$.

\section{Results and discussion}

\section{Dissolution of metal oxide}

The dissolution rate of metal oxide, namely $\mathrm{Co}_{3} \mathrm{O}_{4}$ which represents typically refractory mineral materials, in nitric acid is investigated to elucidate whether combination of microwave and ultrasound have a direct effect upon this type of dissolution reaction. Indeed, the dissolution behaviour of metal oxides and other mineral materials in aqueous solution is of considerable practical importance in the fields as diverse as the extraction of metals from ores, the reprocessing of spent nuclear fuel or the removal of oxide deposits from metal surfaces [24]. Therefore, the understanding of microwave and ultrasound action is determining in terms of reactor design, process optimisation and energy saving.

\section{Kinetic study}

The kinetic study, presented in figure 4 , has been realised following the concentration of cobalt in the solution during the metal oxide dissolution. This study allows a comparison to be made between the classical, microwave and combined MW-US method. In practice, it is difficult to sample from the acidic solution during the digestion procedure because

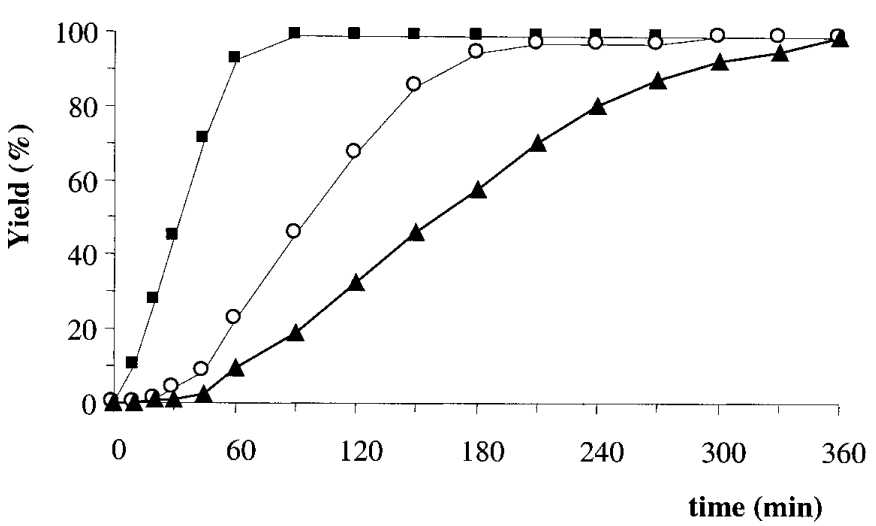

Figure 4. Cobalt recovery as function of time. Combined MW-US (n), microwave (m), and classical method (s ).

of the presence of the fuming nitric acid and various oxidants. In order to rectify this problem, various dissolutions were prepared under the three methods at varying times.

Dissolution (1) was made with nitric acid and hydrogen peroxide under microwave heating. A sample of $0.5 \mathrm{~g}$ of $\mathrm{Co}_{3} \mathrm{O}_{4}$ was introduced into the reactor. In the charring step, $20 \mathrm{~mL}$ of nitric acid $16 \mathrm{M}$ was added and the microwave power was 150 watts. In the oxidative step, $2 \mathrm{~mL}$ of hydrogen peroxide was used and the microwave power was 290 watts for 3 to $10 \mathrm{~min}$.

Dissolution (2) was made with $\mathrm{HNO}_{3}$ and $\mathrm{H}_{2} \mathrm{O}_{2}$ under combined microwave and ultrasound. The monitored reaction conditions of experiment 1 were applied. The ultrasound power was fixed to 150 watts.

Dissolution (3) was made with $\mathrm{HNO}_{3}$ and $\mathrm{H}_{2} \mathrm{O}_{2}$ under classical heating conditions using "Büchi 430". The monitored reaction conditions of experiment 1 were applied.

The dissolution rate with the combined MW-US is six times faster than the classical heating. Under MW-US, the totality of metal oxide is dissolved in only 1 hour, whereas, the classical and the microwave method took respectively 6 and 3 hours. MW heating can creates hot spots at the surface of the metal oxide, by selective heating, enhancing the dissolution's temperature and rate. US favours the cavitation and the erosion of the metal oxide and enhance the mass transfer for the digested cobalt.

After six hours dissolution time, the metal oxide dissolved yields for the three processes became the same. The dissolution kinetic curves differ enormously between the microwave and classical method. Combined MW-US dissolution seem to be first order reactions whereas the conventional method gives a second order kinetic curve.

\section{Effect of microwave and ultrasound power}




\section{Original articles}

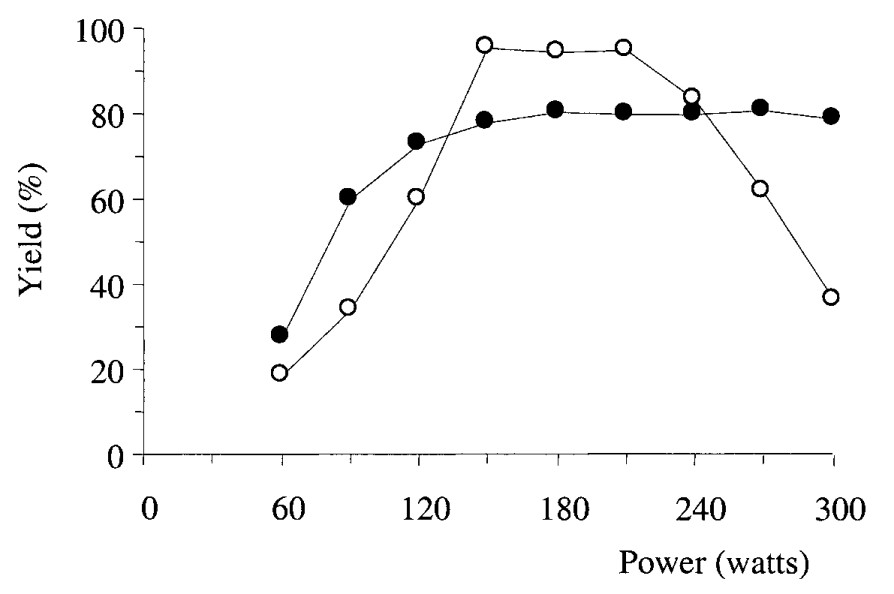

Figure 5. Cobalt recovery as function of MW and US power. 1 MW power (without US, $\mathrm{t}_{\text {total }}=90 \mathrm{~min}$.); $\mathrm{m}$ US power (fixed $P_{\mathrm{MW}}=150 \mathrm{~W}, t_{\text {total }}=60 \mathrm{~min}$.).

Six combined MW-US dissolutions were performed by varying either MW power or US power from 50 to 240 watts. The results are represented in figure 5.

Beyond a microwave power of 125 watts, $80 \%$ of cobalt is recovered by microwave dissolution. At high microwave power, the dissolved cobalt yield is almost the same. The optimum microwave power required is 150 watts. It is sufficient for heating and to avoid the loss of cobalt. Use of the maximal microwave power (300 watts) may damage the magnetron in the long term.

The optimum yield was obtained from a US power of 120 to 170 watts using combined MW-US dissolution. At high US power, the recovered cobalt yield decreases. This is due to the attenuation of US effect or to the loss of cobalt by flash vaporisation. The optimum US power required is 150 watts. It is sufficient for cavitation and dissolution of the solid and to avoid the loss of cobalt.

\section{Digestion of olive oil}

The presence of metals in edible oils occurs through natural contamination and by the introducing during the refining process. Copper is potentially present in oils because of environmental contamination. The presence of metals in oil is undesirable because the metals can facilitate oxidative degradation of the oil and decrease shelf life. The non specific target level for metal contamination is $100 \mathrm{ng} / \mathrm{g}$ [25]. Recent reports regarding the determination of these metals in edible oils at sub-ppm levels have been based on voltammetry [26], atomic absorption [27-28], and atomic emission spectroscopies [29-30]. Most techniques require some type of sample preparation prior to analysis. Sample pretreatement options include off line ashing, solubilisation, and extraction and take several hours of preparations. In this part, we describe an atmospheric pressure MW and MW-US dissolution procedure for edible oils that is compatible with
Table I. Copper recovery yield as function of time (concentration of copper in olive oil $85 \mathrm{ng} / \mathrm{g}$ ).

\begin{tabular}{lccc}
\hline time (min) & $M W-U S$ & $M W$ & Classical \\
\hline 30 & 97.5 & 45.3 & 32.3 \\
45 & 97.2 & 95.8 & 72.3 \\
60 & 98.2 & 98.3 & 97.9 \\
120 & 97.5 & 98.3 & 98.2 \\
\hline
\end{tabular}

coupled plasma atomic emission spectroscopy ICP-AES. The simultaneous effect of MW and US on the digestion of olive oil is also discussed.

The kinetic study, presented in table I, has been realised following the concentration of copper in the solution during olive oil digestion. This study allows a comparison to be made between the classical, microwave and combined MWUS method.

Digestion (1) was made with nitric acid and hydrogen peroxide under microwave heating. A sample of $1 \mathrm{~g}$ of olive oil was introduced into the reactor.

In the charring step, $20 \mathrm{~mL}$ of nitric acid was added and the microwave power was 180 watts for $27 \mathrm{~min}$. In the oxidative step, $3 \mathrm{~mL}$ of hydrogen peroxide was used and the microwave power was 290 watts for $3 \mathrm{~min}$.

Digestion (2) was conducted under classical heating conditions using "Büchi 430". The monitored reaction conditions of experiment 1 were applied.

Digestion (3) was made using combined microwave and ultrasound. The monitored reaction conditions of experiment 1 were applied with a US power of 150 watts.

Under combined MW-US, the totality of copper inside olive oil is digested in only 30 minutes, whereas, the classical and the microwave method took respectively 40 and $60 \mathrm{~min}$.

After two hours digestion time, the copper concentration in the digested solution became the same for the three processes (classical, microwave and combined MW-US).

\section{Conclusions}

The efficiency of the combined microwave and ultrasound apparatus was clearly shown by two examples where decreases in digestion and dissolution time were demonstrated: determination of copper in olive oil, dissolution of refractory mineral material $\mathrm{Co}_{3} \mathrm{O}_{4}$. Table II summarises the most interesting results in terms of efficiency. The simultaneous effect of MW-US is more efficient in heterogeneous media (solid/liquid) than for homogeneous liquid media. Ultrasound favour cavitation occurring near a solid surface. 
Table II. Efficiency of simultaneous MW-US irradiation on the digestion time.

\begin{tabular}{lcc}
\hline Heating system & $\begin{array}{c}\text { Dissolution of } \mathrm{Co}_{3} \mathrm{O}_{4} \\
\text { time }(\mathrm{min}) \\
(\text { Yield }>90 \%)\end{array}$ & $\begin{array}{c}\text { Digestion of olive } \\
\text { oil time } \\
(\text { min) }\end{array}($ Yield $>90 \%)$ \\
\hline 1- Classical & 360 & 60 \\
2- Microwave & 180 & 45 \\
3- Microwave and Ultrasound & 60 & 30 \\
Efficiency (3/1) & 6 & 2 \\
\hline
\end{tabular}

Then, ultrasound generate microjets and shockwaves, resulting in cleaning and erosion of the surface, and fragmentation of the solid. This effect enhances the mass transfer and the digested materials.

Microwave electromagnetic heating is based on the direct absorption of energy by the sample. Then, more energy is collected by the solution, decreasing the digestion time. As a consequence, the coupling MW-US increases the surface constrained mass and heat transfer. Thus a significant improvement in digestion and dissolution can be obtained by using a reactor that combines microwaves and ultrasound.

Simultaneous MW-US irradiation is shown as a new technique for atmospheric pressure digestion and dissolution of solid and liquid samples suitable for chemical and biological analysis. It is expected that the present method could open a new research field "microwave-sonodigestion and extraction".

\section{References}

1. Kingston, H. M.; Haswell, S. J. Microwavem enhanced chemistry: fundamentals, sample preparation and applications, $A$. Chem. Soc., Washington DC, 1998.

2. Kingston, H. M.; Jassie, L. B. Introduction to Microwave Sample Preparation, A. Chem. Soc., Washington DC, 1988.

3. Sinquin, A.; Gorner, T.; Delacherie, E. Analusis 1993, 21, 110.
4. Mathé, D. Analusis 1990, 18, 19.

5. Majors, R. E. $L C-G C, \mathbf{1 9 9 5}, 13,82-94$.

6. Wong M. K,; Gu W.; Ng T. L. Anal. Sci. 1997, 13, 97-102.

7. Kim, Y. D.; Lee, G. H.; Kim, H. S.; Kim, D. S.; Park, K. K. Bull. Korean Chem. Soc. 1994, 15, 786-791.

8. Zlotorzynski, A. Crit. Rev. Anal. Chem. 1995, 25, 43-76.

9. Smith, F. E.; Arsenault, E. A. Talanta 1996, 43, 1207-1268.

10. Capello, J. L.; Lavilla, I.; Bendicho, C. J. Anal. Atom. Spect. 1998, 13, 1285-1290.

11. Chmilenko, F. A.; Baklanova, L. V. J. Anal. Chem. 1997, 52, 1093-1098.

12. Mierzwa, J.; Adeloju, S. B.; Dhinsda, H. S. Anal. Sci. 1997, 13, 189-193.

13. Chemat, F.; Poux, M.; Berlan, J.; Dimartino, J.L. J. M. P. E. E. 1996, 31, 19-22.

14. Maeda, M.; Amemiya, H. N. J. Chem. 1995, 19, 1023-1027.

15. Loupy, A.; Haudrechy, A. Effets de milieu en synthèse organique: des effets de solvants aux méthodes d'activation non classiques, Masson, Paris, 1996.

16. Thuery, J. Les microondes et leurs effets sur la matière, Lavoisier, Paris, 1989.

17. Mason, T. J. Chemistry with ultrasound, Elsvier Applied Science, London, 1990.

18. Suslick, K. S. Ultrasound: chemical and physical and biological effects, VCH, London, 1988.

19. Feinberg, M. H. Analusis 1991, 19, 47-55.

20. Bermond, A.; Ducauze, C. J. Analusis 1991, 19, 64-66.

21. Suard, C. L.; Feinberg, M. H.; Ireland-Ripert, J.; Mourel, M. Analusis 1993, 21, 287-291.

22. Suard, C. L.; Mourel, M.; Didenot, D.; Feinberg, M. H. J. Agri. Food Chem. 1997, 45, 1202-1208.

23. Vasquez, M. J.; Carro, A. M.; Lorenzo, R. A.; Cela, R. Anal. Chem. 1997, 69, 221-225.

24. Joret, L.; Cote, G.; Bauer, D. Hydrometallurgy 1997, 45, 1-12.

25. Allen, L. B.; Siitonen, P. H.; Thompson, H. C. J. A. O. C. S. 1998, 75, 477-481.

26. Wahdat, F.; Hinkel, S.; Deeb, R.; Fresenius, Z. Anal. Chem. 1995, 352, 393-394.

27. Sun, H. J.; J. A. O. C. S. 1989, 66, 549-552.

28. Hendrikse, P. W.; Slikkerveer, F. J.; Zaalberg, J.; Hautefenne Ibid. 1988, 60, 893-900.

29. Ibrahim, H. J. A. O. C. S. 1991, 68, 678-679.

30. Lagha, A. Actes de la troisième conférence maghrébine de génie des procédés, Tamanrasset (Algeria), 1998, 172-175. 